\title{
Erratum: dimensionally regularized Polyakov loop correlators in hot QCD
}

\author{
Y. Burnier, ${ }^{a, 1,2}$ M. Laine ${ }^{a, 1,3}$ and M. Vepsäläinen ${ }^{b}$ \\ ${ }^{a}$ Faculty of Physics, University of Bielefeld, \\ D-33501, Bielefeld, Germany \\ ${ }^{b}$ Department of Physics, University of Helsinki, \\ P.O. Box 64, FI-00014, Helsinki, Finland \\ E-mail: yburnier@physik.uni-bielefed.de, \\ laine@physik.uni-bielefed.de, mtvespal@pcu.helsinki.fi
}

ERRATUM TO: JHEP01(2010)054

\section{ArXiv EPRINT: 0911.3480}

In the analysis of section 5 several Fourier transforms appear (eqs. (5.4), (5.13), (A.19)) which, taken literally, are ambiguous, because of a pole on the integration contour. Whereas the first two were defined in the sense of a principal value, the last one was defined in the sense of a right value (leaving poles to the right of the integration contour). This leads to an inconsistency when the results are summed together. Re-evaluating the last case as a principal value we get

$$
\begin{aligned}
\sum_{q_{n}^{\prime}} \int_{\mathbf{q}} \mathbb{P}\left(\frac{e^{i q_{z} r}-1}{q_{z}^{2} Q^{2}}\right)= & \frac{\beta}{8 \pi^{2}}\left[\frac{1}{\epsilon}+L_{\mathrm{b}}+2 \ln \left(1-e^{-2 \pi T r}\right)+4 \pi T r \sum_{n=1}^{\infty} E_{1}(2 \pi T r n)\right] \\
& +\frac{r}{8 \pi}\left[\frac{1}{\epsilon}+L_{\mathrm{b}}+2\left(\ln 4 \pi-\gamma_{\mathrm{E}}\right)\right] .
\end{aligned}
$$

The term on the 2nd row, which was absent from the original analysis, acts as a "counterterm" to the long-distance contribution in eq. (5.13); more specifically, it cancels the divergences in eqs. (5.26), (5.28), (5.29). Consequently, provided that the overall divergences of eqs. (5.24), (5.25) can also be successfully taken care of, as has recently been proposed in ref. [1], the quantity $\bar{m}_{\mathrm{E}}$ defined in eq. (5.27) could be finite. We thank the authors of ref. [1] for drawing our attention to the inconsistency.

\section{References}

[1] M. Berwein, N. Brambilla, J. Ghiglieri and A. Vairo, Renormalization of the cyclic Wilson loop, arXiv: 1212.4413 [INSPIRE].

\footnotetext{
${ }^{1}$ Current address: Institute for Theoretical Physics, Albert Einstein Center, University of Bern, Sidlerstrasse 5, CH-3012 Bern, Switzerland

2yburnier@itp.unibe.ch

${ }^{3}$ laine@itp.unibe.ch
} 